\title{
Non-destructive testing procedure for porosity determination in carbon fibre reinforced plastics using pulsed thermography
}

\author{
by G. Mayr*, K.-H. Gresslehner* and G. Hendorfer*
}

*University of Applied Sciences Upper Austria, School of Engineering, Stelzhamerstrasse 23, 4600 Wels, Austria, guenther.mayr@fh-wels.at

\begin{abstract}
The aim of this work was to develop a procedure for the application of pulsed thermography as a quantitative method for porosity determination in carbon fibre reinforced plastics (CFRP). Mathematical models are used to link the microstructural properties with global thermal properties which are the diffusion time and the thermal diffusivity. These detailed mathematical models, based on the effective medium theory, can be simplified for practical applications in the industry. It is possible to calculate a porosity image by the measurement of the thermal diffusion time. The predicted porosity image matches the measured porosity image based on cone beam X-ray computed tomography (XCT) reference measurement quite well.
\end{abstract}

\section{Introduction}

In the industry, especially in the aviation industry non-destructive testing (NDT) of components is one of the most critical aspects in design and manufacturing. The requirement of damage-tolerant design needs NDT methods which are capable of finding flaws and characterizing defect sizes. A critical and unavoidable defect type in carbon fibre reinforced plastics (CFRP) is the inclusion of gas-filled voids named as porosity [1]. Matrix-dominated material properties, such as transverse tensile strength and interlaminar shear strength, decrease with increasing porosity [2, 3]. In the aerospace industry a porosity level of $2.5 \%$ is defined as a general level of acceptance for most safety-critical CFRP components. The quantitative characterization of the material and microstructural parameters of porous structures is therefore essential for the wide use of CFRP in safety-relevant aviation components. The aim of this work was to develop a test procedure for the practical application of pulsed thermography as a quantitative method for porosity determination in CFRP components.

Early studies showed the great potential of the NDT method pulsed thermography to detect and localize porosity in CFRP $[4,5]$. Pulsed thermography allows the spatially resolved determination of the porosity dependent thermophysical material properties such as the thermal diffusivity or thermal effusivity. Experimental studies from Hendorfer [6] and Zalameda [7] show that the thermal diffusivity decreases nearly linearly with increasing porosity. A comparison of ultrasonic C-scan images, thermal diffusivity and thermal effusivity images derived from experiments on aerospace components are published by Grinzato et al. [8]. Ringermacher developed a so-called "dethermalization theory" for the description of the porosity dependence of the effective volumetric heat capacity and effective thermal conductivity [9]. The dethermalization theory is derived from an effective medium theory developed by Mori-Tanaka [10, 11]. The dethermalization factor $\eta$ takes into account the influence of the pore shape on the heat flux and therefore on the effective thermal conductivity. The dethermalization factor is calculated from the aspect ratio of the axis of the representative (averaged) pore shape. Cone beam X-ray computed tomography (XCT) data can be used for the characterisation of the microstructure and especially of the pore shape $[12,13]$. Results of the former experimental studies allow the validation of the dethermalization theory on experimental results obtained from calibrated porosity coupons [14] and from numerical studies based on real and artificial pore distributions [15].

Notably for the practical use in industrial applications these approximations should be simplified. For low porosity levels the models can be linearized and the porosity can be calculated from analytical expressions based on the measured thermal properties. The aim of this work is therefore to describe a procedure for the application of pulsed thermography as a quantitative method for the determination of the porosity in CFRP.

\section{Testing procedure for porosity determination} in two fields:

The non-destructive testing procedure for porosity determination in CFRP using pulsed thermography is divided

- $\quad$ Step 1: Calibration procedure for the determination of the dethermalization factors

- Step 2: Measurement procedure for pulsed thermography 
The main activities in both steps are described in the flow chart given in figure 1. The calibration procedure has to be carried out for every specific material system (unidirectional or fabric) and the measurement procedure for every part which has to be tested.

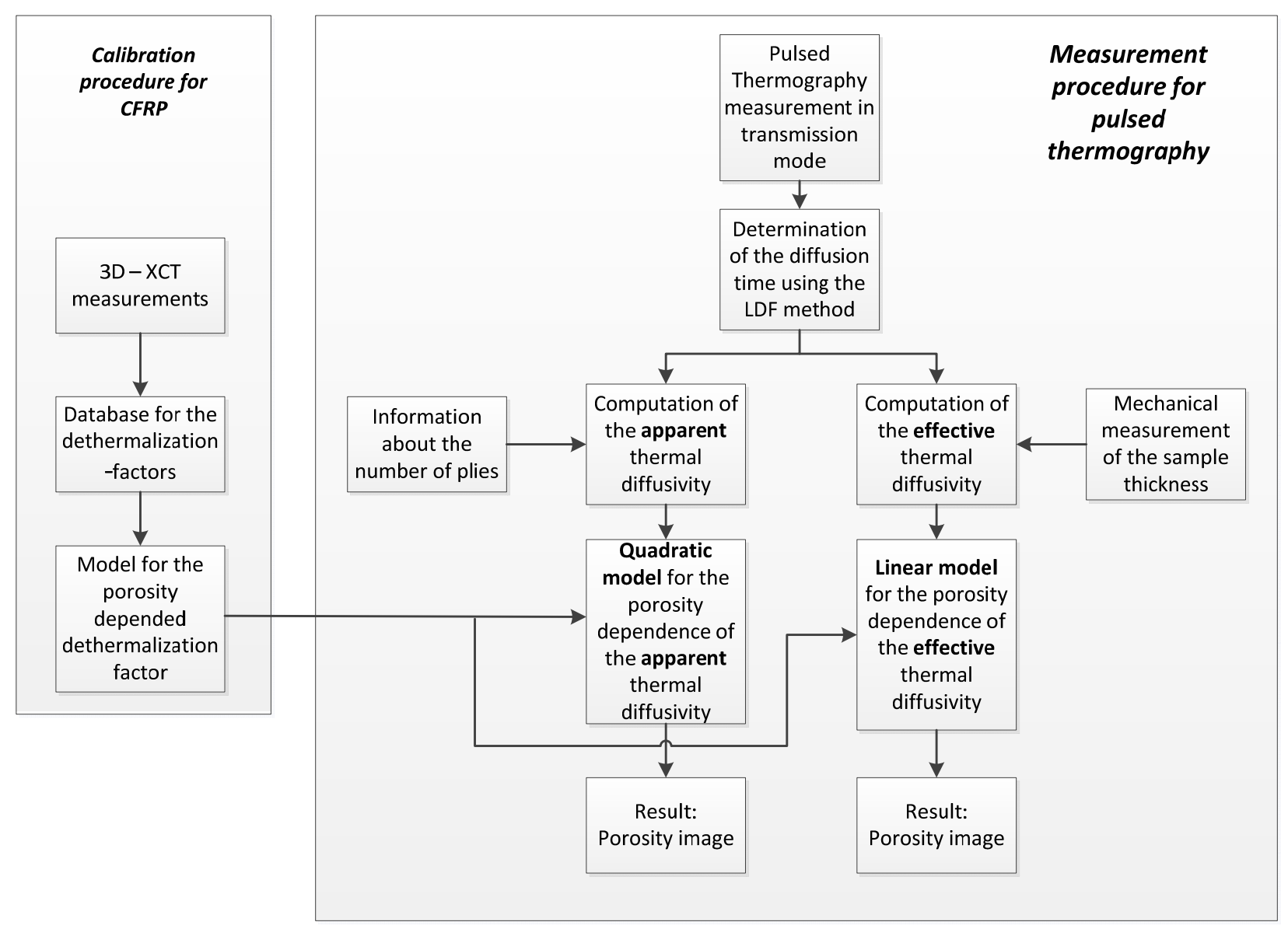

Fig. 1. Flow chart of the test procedures for porosity determination in CFRP using pulsed thermography.

\subsection{Calibration procedure for CFRP}

For the determination of the effective thermal conductivity $k_{\text {eff }}$ the aspect ratio $m$ of the representative pore and the dethermalization factor $\eta$ must to be taken into account. To model the dethermalization factor $\eta$ the actual voids are replaced by oblate spheroids with the same axis lengths as the actual one. The major axis of the voids is aligned parallel to the sample surface. The dethermalization factor $\eta$ of an oblate spheroid can be calculated by the following expression given in [9]:

$$
\eta=\frac{m^{2}}{m^{2}-1}-\frac{m^{2}}{\left(m^{2}-1\right)^{3 / 2}} \arcsin \left(\frac{\left(m^{2}-1\right)^{1 / 2}}{m}\right)
$$

where $m$ is defined as the ratio of the long axis length (a) to the short axis length (b) of an oblate spheroid $(m=a / b>1)$. In this work the mean aspect ratios and the corresponding dethermalization factors were evaluated for 13 different porosity coupons with porosities in the range of $0 \%$ to $10 \%$. In figure 2 the dethermalization factors for the different porosity coupons are plotted versus the global (macroscopic) porosity. The global porosity describes the whole void content over the test specimen and is derived by cone beam XCT measurements. As can be seen from figure 2 the dependence of the dethermalization factor $\eta$ from the porosity $\Phi$ looks like a capacitor charging curve. The trial function is given in Eq. 2 and the fitting of the measured data was carried out by a non-linear least square method: 


$$
\eta=\eta_{0}+\left(\eta_{\max }-\eta_{0}\right) \cdot\left(1-e^{-\Phi / \Phi_{0}}\right)
$$

where $\eta_{\max }=0.72, \eta_{0}=0.50$ and $\Phi_{0}=0.026 . \Phi_{0}$ is the so called characteristic porosity. As mentioned by Stone and Clarke [16] the larger pores are flattened and elongated due to the limitation of space between the fibre plies. At low porosity levels the voids have roughly circular cross-sections with an aspect ratio of 1.8 and a dethermalization factor of 0.5 . At high porosity values the dethermalization factor converges to 0.72 .

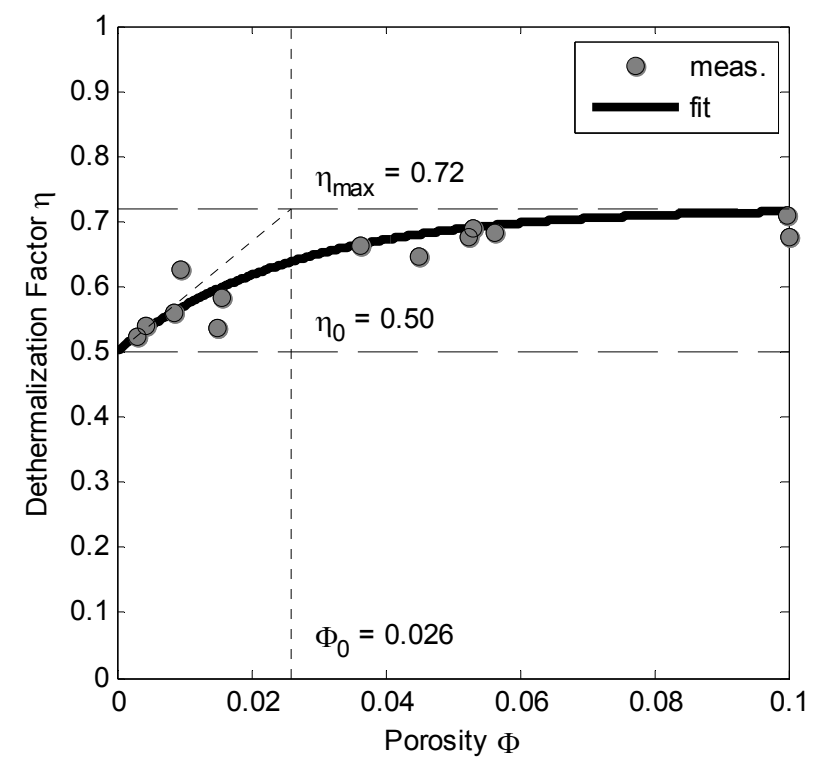

Fig. 2. Dependence of the dethermalization factor on the porosity. The circles indicate measurements and the solid line are the calculated values given by Eq. 2.

\subsection{Model for the porosity dependence of the thermal diffusivity}

The mathematical description of the effective thermal diffusivity (Eq. 3) is given by Mori-Tanka [10, 11]:

$$
\alpha_{\mathrm{eff}}=\frac{k_{\mathrm{eff}}}{(\rho \cdot c)_{\mathrm{eff}}}=\frac{k_{\mathrm{m}}+\Phi \cdot\left(k_{\mathrm{p}}-k_{\mathrm{m}}\right) \cdot \frac{k_{\mathrm{m}}}{k_{\mathrm{m}}+\eta \cdot\left(k_{\mathrm{p}}-k_{\mathrm{m}}\right)+\Phi \cdot \eta \cdot\left(k_{\mathrm{m}}-k_{\mathrm{p}}\right)}}{(\rho \cdot c)_{\mathrm{p}} \cdot \Phi+(\rho \cdot c)_{\mathrm{m}} \cdot(1-\Phi)}
$$

where $k_{\text {eff }}$ is the effective thermal conductivity and $(\rho c)_{\text {eff }}$ is the mixture rule for the effective volumetric heat capacities. The subscripts $m$ and $p$ denote the matrix (carbon fibre and epoxy resin) and the pores, respectively. The model requires the thermophysical properties of the matrix and pore phase which are given in table 1 . This model is validated for porosity levels less than $10 \%$. An additional requirement for the use of this model is, that the pores are randomly distributed, unidirectional aligned parallel to the layer and not connected by each other.

Table 1. Thermophysical properties of the CFRP constituents used for the effective medium approximation [17, 18].

\begin{tabular}{llccc}
\hline Property & & Unit & Matrix & Pore \\
\hline Thermal conductivity & $k$ & $\mathrm{~W} /(\mathrm{m} \mathrm{K})$ & 0.7 & 0.026 \\
Density & $\rho$ & $\mathrm{kg} / \mathrm{m}^{3}$ & 1570 & 1.2 \\
Heat capacity & $c$ & $\mathrm{~J} /(\mathrm{kg} \mathrm{K})$ & 1200 & 1000 \\
\hline Thermal diffusivity & $\alpha$ & $\mathrm{m}^{2} / \mathrm{s}$ & $3.72 \cdot 10^{-1}$ & $2.17 \cdot 10^{-5}$ \\
\hline
\end{tabular}

Due to the fact, that in CFRP $k_{\mathrm{p}} \ll k_{\mathrm{m}},(\rho c)_{\mathrm{p}} \ll(\rho c)_{\mathrm{m}}$ and $\eta$ is of the order of 1 , Eq. 3 can linearized for porosities in the range of $\Phi=0$ to 0.1 : 


$$
\alpha_{\mathrm{eff}}=\alpha_{0}+\alpha_{1} \Phi \ldots \text { linear model }
$$

where $\alpha_{0}$ is the thermal diffusivity of a porosity-free specimen and $\alpha_{1}$ is the sensitivity coefficient which represents the relative change of the thermal diffusivity due to the relative change of the global porosity. As shown in figure 3(a) the linearization of $\alpha_{\text {eff }}$ versus $\Phi$ (Eq. 4) fits very well the Mori-Tanaka Model (Eq. 3) in the range of $\Phi=0$ to 0.1 . However, the porosity is defined as the ratio of the volume of the pores $V_{p}$ divided by the total volume of the material and voids $V=V_{m}+V_{p}$ by following equation $\Phi=V_{p} / V$. Due to the orthotropic stiffness of the fabric CFRP the specimen volume increases in one direction normal to the layers which can be described by

$$
L=L_{0} \cdot \frac{1}{1-\Phi}
$$

where $L_{0}$ is the nominal sample thickness defined as $L(\Phi=0)=L_{0}=N I_{\mathrm{x}}$, where $N$ is the number of plies and $I_{\mathrm{x}}$ the nominal thickness of a ply.

In pulsed thermography measurements the determined thermal diffusivity $\alpha$ depends both on the material parameters and the porosity as well as on the thickness of the specimen. For the exact determination of the effective thermal diffusivity it is necessary to know the thickness of the specimen. A method for the determination of the porosity of CFRP whose actual thickness is not known is to determine the so-called apparent thermal diffusivity $\alpha_{\text {app. That means }}$ that in this case we are not able to determine the actual value of the specimen thickness $L(\Phi)$ by mechanical or other measurement methods. Therefore, we only have to know the number of plies and the nominal ply thickness in the tested area. The apparent thermal diffusivity can be expressed in the following way by taking into account Eq. 5

$$
\alpha_{\text {app }}=\alpha_{\text {eff }}\left(\frac{L_{0}}{L}\right)^{2}=\left(\alpha_{0}+\alpha_{1} \Phi\right) \cdot(1-\Phi)^{2}
$$

From Eq. 6a it is apparent that after multiplying both terms a term on the right hand side containing $\Phi^{3}$ occurs. Due to the fact, that $\alpha_{0}$ and $\alpha_{1}$ are in the same order and $\Phi$ was chosen less than 0.1 this $\Phi^{3}$ term can be neglected. Therefore, $\alpha_{\text {app }}$ is given by

$$
\alpha_{\text {app }}=\alpha_{0}+\Phi \cdot\left(\alpha_{1}-2 \alpha_{0}\right)+\Phi^{2} \cdot\left(\alpha_{0}-2 \alpha_{1}\right) \ldots \text { quadratic model }
$$

Figure 3(b) shows the calculated apparent thermal diffusivity versus porosity based on the exact expression given in Eq. 3 and Eq. 5 and the approximation given in Eq. 6b. As one can see, there is an excellent agreement in the porosity range of 0 to 0.1 .

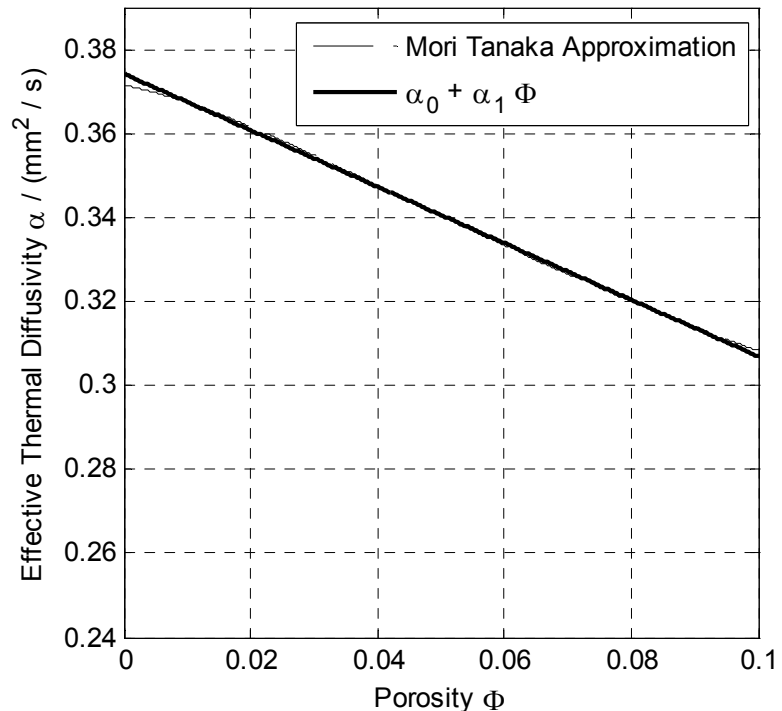

(a) Effective thermal diffusivity

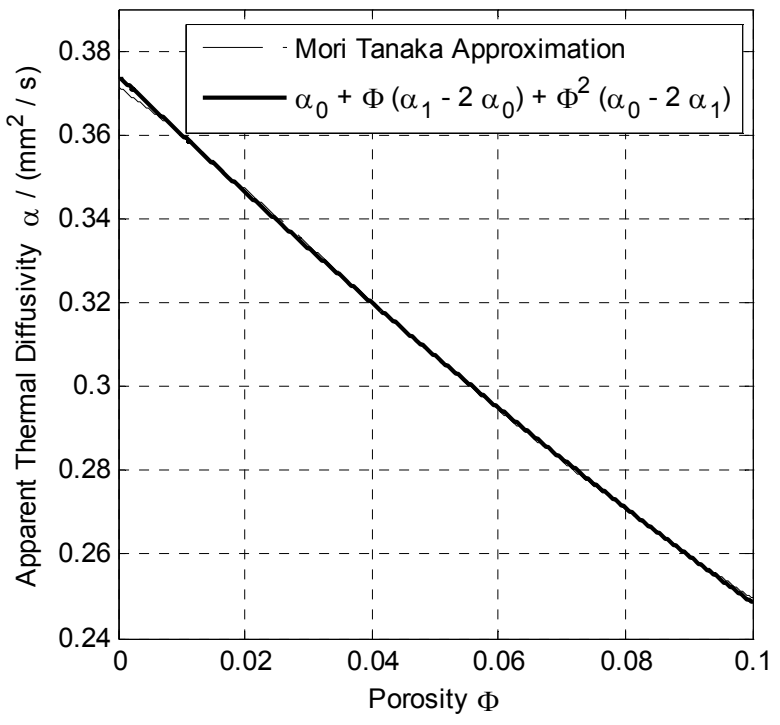

(b) Apparent thermal diffusivity

Fig. 3. Comparison of the Mori-Tanaka model with a linearized model for the (a) effective and (b) apparent thermal diffusivity for porosities in the range of 0 to 0.1 . 


\subsection{1/qirt.2016.081}

From Eq. 4 the porosity can be calculated straightforward from the effective thermal diffusivity and the fit parameters $\alpha_{0}$ and $\alpha_{1}$ :

$$
\Phi=\frac{\alpha_{\mathrm{eff}}-\alpha_{0}}{\alpha_{1}}
$$

A similar approach for the approximated apparent thermal diffusivity can be obtained by the quadratic model (Eq. 6b)

$$
\begin{aligned}
& \Phi=\frac{1}{2} \cdot\left(\frac{2-A}{2 A-1}\right)\left(\sqrt{1+4 \frac{(2 A-1)(1-B)}{(2-A)^{2}}}-1\right) \\
& A=\frac{\alpha_{1}}{\alpha_{0}} \\
& B=\frac{\alpha_{\text {app }}}{\alpha_{0}}<1
\end{aligned}
$$

with the same fit parameters $\alpha_{0}$ and $\alpha_{1}$ as used in Eq. 7, where $\alpha_{0}=0.374 \mathrm{~mm}^{2} / \mathrm{s}$ and $\alpha_{1}=-0.673 \mathrm{~mm}^{2} / \mathrm{s}$. These mathematical models are easy to handle and can be used in practice for an exact prediction of the porosity in CFRP based on the measured effective or apparent thermal diffusivity.

\subsection{Measurement procedure for pulsed thermography}

The evaluation of thermo-physical material properties is of particular interest in pulsed thermography. The quantitative determination of the thermal diffusivity shows advantages in terms of higher resistance against undesired effects such as errant reflections of the surrounding, emissivity variations or environmental conditions. Former studies have shown that only measurements in a transmission mode allow a precise quantitative determination of the thermal diffusivity which is sufficiently accurate for porosity determination [14]. In figure 4 the experimental setup of optical excited pulsed thermography in transmission mode is shown. As heat source a flash lamp with an energy deposition of 6 $\mathrm{kJ}$ and a pulse duration of $2 \mathrm{~ms}$ is used. The temperature measurement was carried out with an infrared (IR) camera equipped with an indium antimonide $(\mathrm{InSb})$ detector. The IR camera allows the observation of the temperature increase at the back side of the specimen $(z=L)$ after a short pulse excitation at the front side $(z=0)$. This cooled $320 \times 256$ pixel FPA camera has a thermal sensitivity of $20 \mathrm{mK}$ in a spectral range of $3-5 \mu \mathrm{m}$. A frame rate of $50 \mathrm{~Hz}$ was chosen.

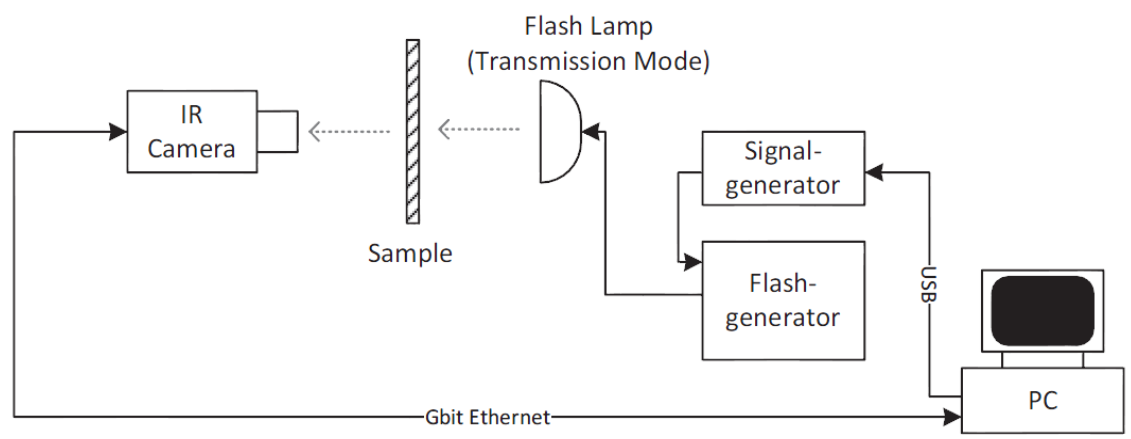

Fig. 4. Experimental setup of optical excited pulsed thermography in transmission mode for the quantitative determination of the thermal diffusivity.

Several methods have been developed to compute the thermal diffusivity in transmission mode [19]. In this study a procedure was applied to measure the observation time $t_{\mathrm{obs}}$, which is closely related to the standardized Parker's method [20]. The linear diffusivity fitting method (LDF) enables a more reliable and precise prediction of $t_{\mathrm{obs}}$ especially for low Signal to Noise ratios. The change of the measured observation time $\left(t_{\mathrm{obs}}=\mathrm{L}^{2} / \alpha\right)$ caused by the presence of voids and increasing specimen thickness can be used for the non-destructive porosity prediction. The effective thermal 
diffusivity is derived from the measured observation time $t_{\mathrm{obs}}$ using the actual sample thickness $L(\Phi)$ and the apparent thermal diffusivity from the nominal sample thickness $L(\Phi=0)=L_{0}$ :

$$
\alpha_{\text {eff }}=\frac{L(\Phi)^{2}}{t_{\text {obs }}} \quad \text { and } \quad \alpha_{\text {app }}=\frac{L_{0}^{2}}{t_{\text {obs }}}
$$

The prerequisites under which the effective thermal diffusivity can be calculated is: (i) knowledge of the actual sample thickness for each point of the sample which means, that it must be possible to measure the sample thickness at each point with mechanical or other measurement instruments. The prerequisites under which the apparent thermal diffusivity can be calculated are: (i) knowledge of the nominal sample thickness (number of plies) and (ii) a one-dimensional (1D) volume expansion in the case of increasing porosity.

\section{Experimental verification}

Two sets of 10 and 20 plies woven-fabric CFRP were investigated to verify experimentally the procedures for porosity determination. The CFRP coupons were fabricated in an autoclave process with different pressures and temperatures to contain various amounts of void contents, from $0 \%$ to $10 \%$ porosity. Each of the 32 coupons has a lateral size of $(40 \times 20) \mathrm{mm}^{2}$ and the nominal ply thickness of the used prepreg is $I_{x}=0.216 \mathrm{~mm}$.

In figure 5(a) the mechanical measured coupon thickness is plotted versus the porosity obtained by cone beam XCT measurements. The thickness of the porous specimens rises nearly linearly by increasing the porosity. The measured results are in complete agreement with the theoretical prediction (Eq. 5). The measured observation time as a function of the porosity is shown in figure 5(b). The results of samples of different number of plies are relatively consistent, with an increase in the observation time produced by an increase in porosity. The observation times are obtained by averaging the local values over a spatial domain $(20 \times 20) \mathrm{mm}^{2}$. A standard deviation of the measured observation time using pulsed thermography in transmission mode of $\sigma_{\text {tobs }}=0.6 \%$ was derived from 10 measurements.

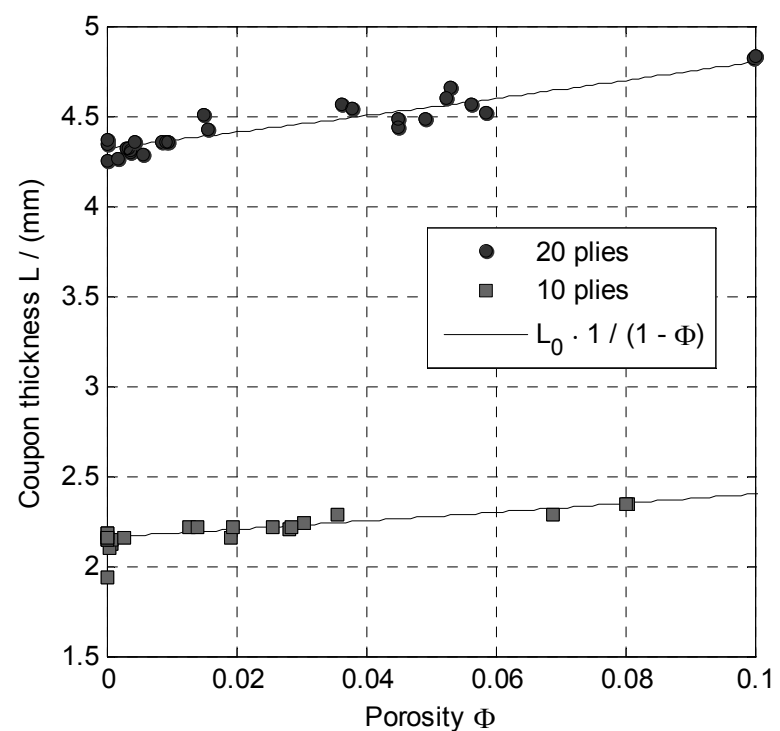

(a) Coupon thickness versus porosity

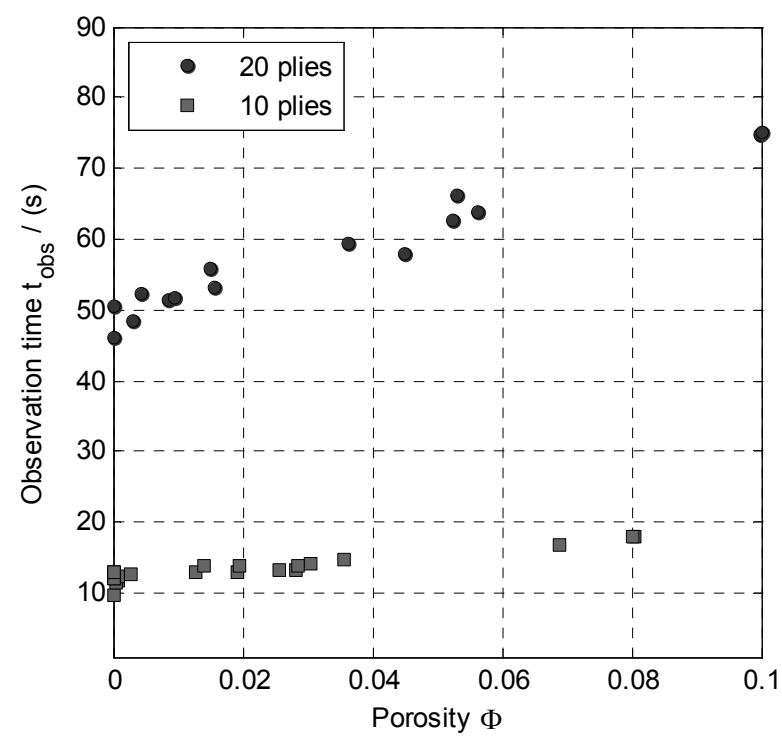

(b) Observation time versus porosity

Fig. 5. A plot of the mechanical measured coupon thickness versus porosity (a) and the observation time values in transmission mode of 10 and 20 plies porosity samples versus porosity obtained by XCT (b).

The validity of the linear and quadratic model of thermal diffusivity modeling is basically confirmed by the comparatively small deviation of the experimental data from the predicted thermal diffusivity values (Fig. 6(a) and 7(a)). For the prediction of the thermal diffusivity the thermophysical properties given in table 1 were used. As can be seen from the figures the agreement between the models and measurements is better for the apparent thermal diffusivity. The figures $6(\mathrm{~b})$ and 7 (b) indicate that the linear and quadratic expressions of Eq. 7 and Eq. 8 can be used to calculate the porosity from the measured effective and apparent thermal diffusivity. The prediction accuracies are slightly different. The absolute error in the determination of the void content is about $0.9 \%$ porosity for the linear model-based prediction on the effective thermal diffusivity and $0.6 \%$ porosity for the quadratic model-based prediction on the apparent thermal diffusivity. 


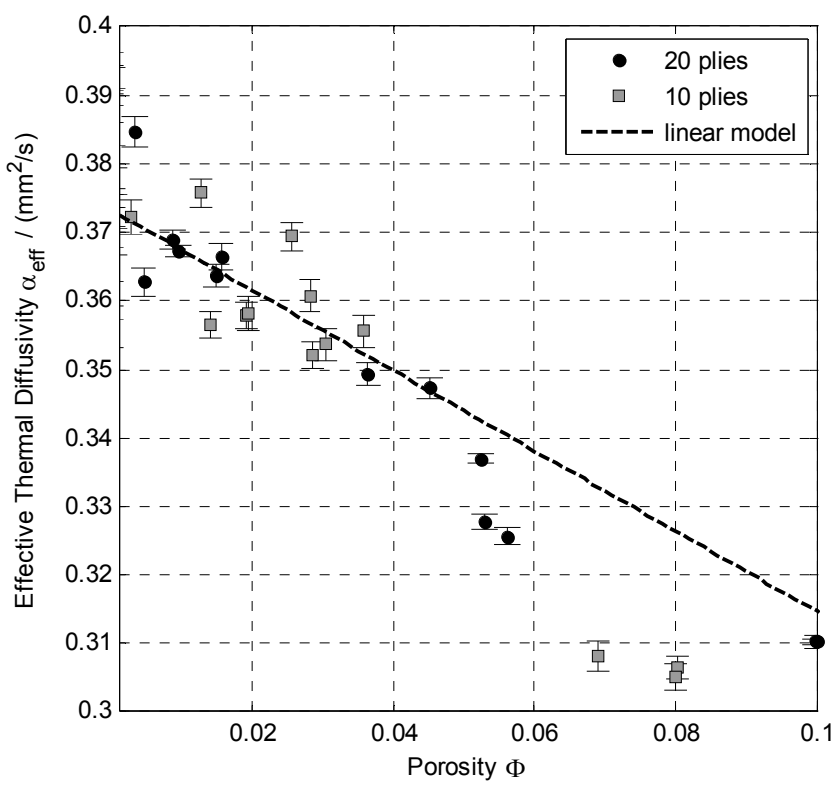

(a) Effective thermal diffusivity versus porosity

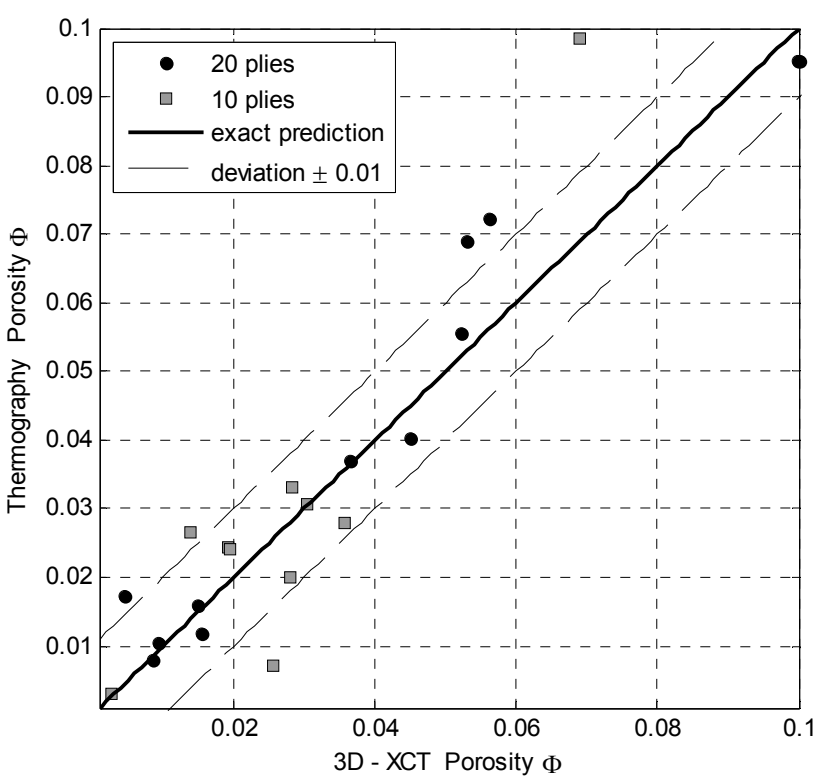

(b) Porosity derived with thermography versus porosity derived by XCT

Fig. 6. Effective thermal diffusivity: (a) A plot of the effective thermal diffusivity according to measurement results of 10 and 20 plies CFRP and the linearized model (Eq. 7) versus porosity. (b) A plot of the predicted porosity levels based on pulsed thermography and Eq. 7 in transmission mode versus the exact porosity obtained by XCT measurements.

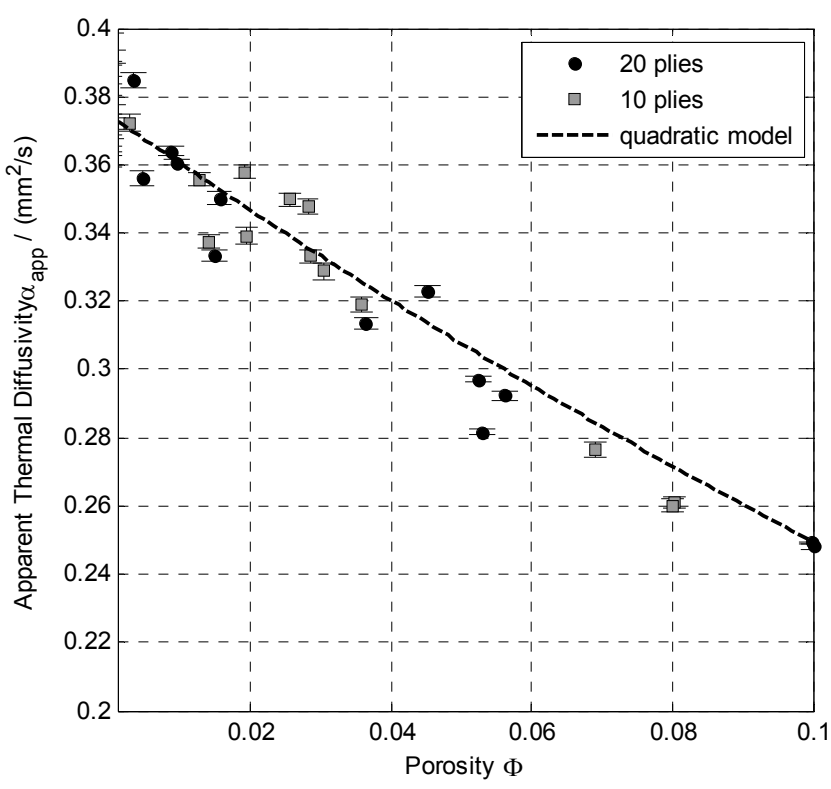

(a) Apparent thermal diffusivity versus porosity

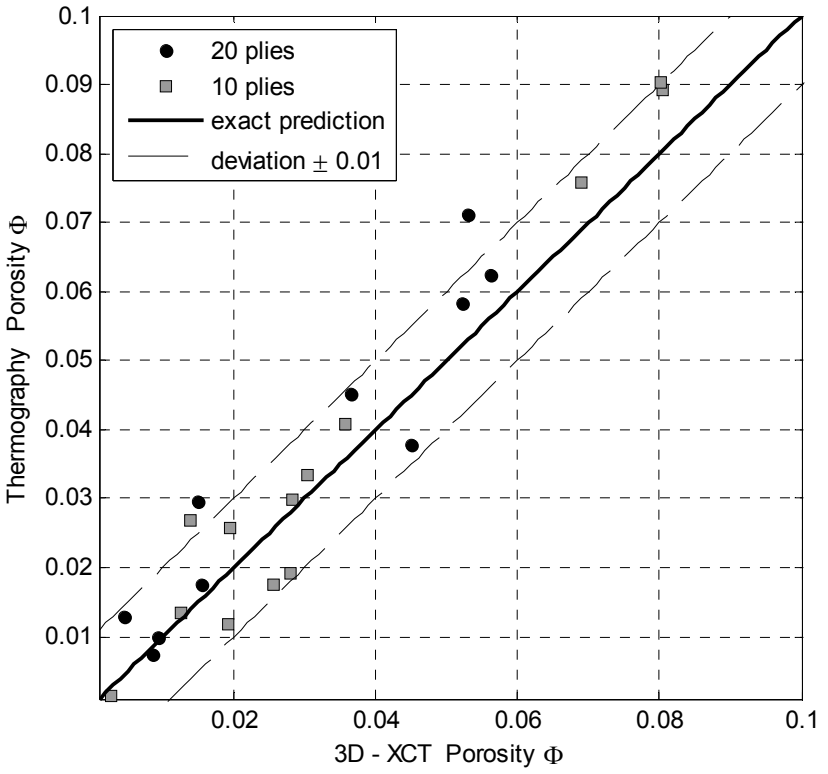

(b) Porosity derived with thermography versus porosity derived by XCT

Fig. 7 Apparent thermal diffusivity: (a) A plot of the apparent thermal diffusivity according to measurement results of 10 and 20 plies CFRP and the simplified quadratic model (Eq. 8) versus porosity. (b) A plot of the predicted porosity levels based on pulsed thermography and Eq. 8 in transmission mode versus the exact porosity obtained by XCT measurements. 
The spatially resolved porosity determination is demonstrated on a CFRP coupon with 10 plies and a global porosity of $\Phi=6.9 \%$. The porosity coupon with a size of $(40 \times 20) \mathrm{mm}^{2}$ was investigated by pulsed thermography in transmission mode and cone beam XCT. The thermography measurement was carried out with a spatially resolution of $130 \mu \mathrm{m} /$ pixel and the XCT measurement with $10 \mu \mathrm{m} /$ voxel. Figure 8(a) shows a porosity image, which was derived by pulsed thermography measurement of the apparent thermal diffusivity and Eq. 8 for the porosity determination. The red areas indicate regions with higher porosity and the blue areas show regions with low porosity. It can be seen that there is a strong porosity gradient in the CFRP coupon from the upper left to the lower right corner. The inhomogeneous porosity content derive from the autoclave process where different pressures were applied. The XCT measurement window (18 $\mathrm{x}$ 18) $\mathrm{mm}^{2}$ is denoted with a grey dotted rectangle. The original porosity data derived from XCT measurement is plotted in figure $8(\mathrm{~b})$. With a spatial resolution of $(10 \times 10) \mu \mathrm{m}^{2}$ the pore and matrix volumes are summarized in $z$ - direction and plotted as a porosity value in a colour scaled image. The XCT image shows that in the $x-y$ plane the pores aren't randomly distributed. There is a systematically arrangement due to the fibre placement. Nevertheless, the XCT results show the same gradient as the thermography results. The spatially high resolved XCT image isn't comparable to the pulsed thermography image because the diffusion of heat blurred the porosity image. For the purpose of comparing the original XCT data is also blurred by a mean filter with a cubic size of $(2 \times 2 \times 2) \mathrm{mm}$ (Fig 8(c)) which is the size of the representative elementary volume (REV). Additionally, the porosity image derived by thermography is compared the averaged XCT data by using two line profiles of porosity. The model-based predicted porosity values using pulsed thermography match the XCT data quite well. As a result the NDT method pulsed thermography can be validated for a new method for the quantitative porosity determination in CFRP.

(a) Porosity Image (Pulsed Thermography)
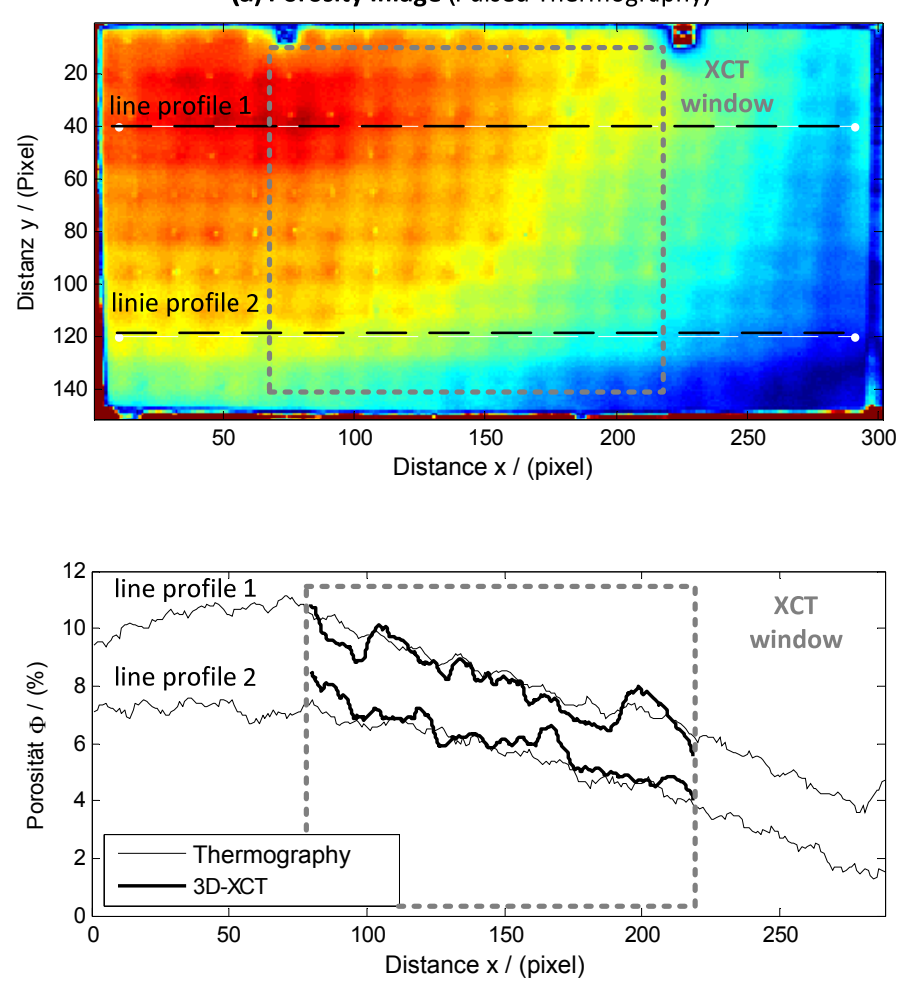

(b) Porosity Image (XCT) original data

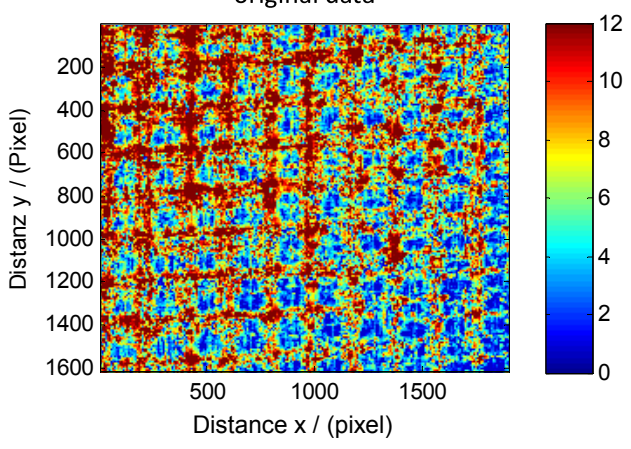

(c) Porosity Image (XCT)

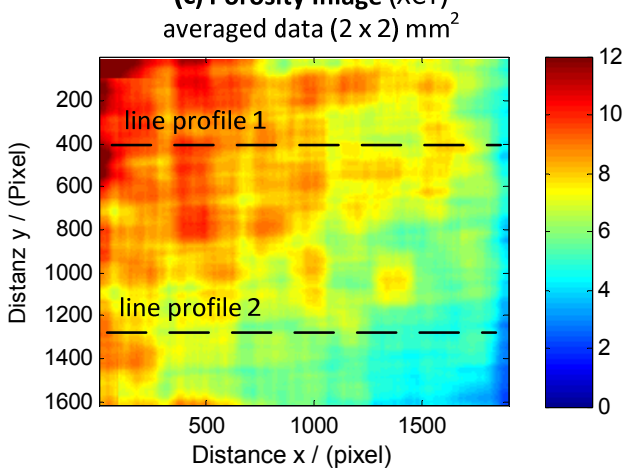

Fig. 8 Results of the spatially resolved porosity determination carried out on a flat CFRP coupon with 10 plies and a global porosity of $\Phi=6.9 \%$. The colour-scaled porosity images show a comparison between pulsed

thermography (a), the original cone beam XCT data (b) and the volume averaged XCT data over a cube with an edge length of $2 \mathrm{~mm}$ (c). The line profiles show a quantitative comparison between pulsed thermography and cone beam XCT.

\section{Conclusion}

A NDT procedure has been developed which allows the application of pulsed thermography for the quantitative determination of porosity in CFRP. A linearized model to calculate the effective thermal diffusivity requires only two parameters $\alpha_{0}$ and $\alpha_{1}$. The porosity free thermal diffusivity $\alpha_{0}$ can be measured on a void free CFRP coupon. The sensitivity coefficient $\alpha_{1}$ is derived from the Mori-Tanaka model. The required dethermalization factor is determined by 


\subsection{1/qirt.2016.081}

cone beam XCT measurements and a curve fitting procedure. Comparisons between calculated and experimental data show an excellent agreement for a porosity from $\Phi=0$ to 0.1 . The models for the effective and apparent thermal diffusivity may then be used to determine the global porosity based on pulsed thermography measurements data. The accuracy of porosity determination based on pulsed thermography is comparable to ultrasonic testing with the advantages of a fast and non-contact measurement procedure. In further studies, the parameters $\alpha_{0}$ and $\alpha_{1}$ should be determined for unidirectional prepreg and resin transfer moulding materials.

\section{Acknowledgment}

This work was financially supported by the TAKE OFF program of the Bundesministerium für Verkehr, Innovation und Technologie (BMVIT). Furthermore, we wish to thank our cooperation partner FACC Operations $\mathrm{GmbH}$.

\section{REFERENCES}

[1] Campbell F.C., Structural Composite Materials. Ohio: ASM International; 2010.

[2] Ghiorse S. R., "Effect of void content on the mechanical properties of carbon/epoxy laminates", SAMPE Quaterly, vol. 24(2), pp. 54-59, 1993.

[3] Oliver P., Cottu J. P., Ferret B., "Effects of cure cycle pressure and voids on some mechanical properties of carbon/epoxy laminates", Composites, vol. 26(2), pp. 509-515, 1995.

[4] Connolly M. P., "The measurement of porosity in composite materials using infrared thermography". Journal of Reinforced Plastics and Composites, vol. 11(12), pp. 1367-1375, 1992.

[5] Heath D. M., Winfree W. P., "Thermal diffusivity measurement in carbon-carbon composites", Proceedings of the Review of Progress in QNDE, vol. 88, New York: Plenum Press, pp. 1613-1619, 1989.

[6] Hendorfer G., Mayr G., Zauner G., Haslhofer M., Pree R., "Quantitative determination of porosity by active thermography“, Proceedings of the Review of Progress in QNDE, vol. 26, AIP, pp. 702-708, 2007.

[7] Zalameda J.N., Winfree W.P., "Thermal diffusivity measurements on composite porosity samples", Review of Progress in Quantitative Nondestructive Evaluation, vol. 9, 1990.

[8] Grinzato E.G., Marinetti S., Bison P.G., "NDE of porosity in CFRP by multiple thermographic techniques", Proc. SPIE 4710, Therosense XXIV, Orlando, 2002.

[9] Ringermacher H. I., Howard D.R. and Gilmore R.S., "Discriminating porosity in composites using thermal depth imaging", AIP conference proceedings, vol. 615(1); 2002. p. 528-535.

[10] Mori T., Tanaka K., "Average stress in matrix and average elastic energy of materials with misfitting inclusions", Acta Metallurgica, vol. 21(5), pp. 571-574, 1973.

[11] Torquato S., Random heterogeneous materials. London: Chapman \& Hall; 2002.

[12] Kastner J., Plank B., Salaberger D., Sekelja J., "Defect and porosity determination of fibre reinforced polymers by $x$-ray computed tomography", $2^{\text {nd }}$ International Symposium on NDT in Aerospace, p. 12, 2010.

[13] Reh A., Plank B., Kastner J., Groeller E., Heinzl C., "Porosity maps - Interactive exploration and visual analysis of porosity in carbon fiber reinforced polymers", Eurographics Conference on Visualization (EuroVis), vol. 31(3), 2012.

[14] Mayr G., Plank B., Gruber J., Sekelja J., Hendorfer G., "Quantitative evaluation of the effective thermal diffusivity for model-based porosity prediction in CFRP". Quantitative InfraRed Thermography Journal. DOI: 10.1080/17686733.2015.1093310, 2015.

[15] Mayr G., Gruber J., Hendorfer G., "Analytical and numerical computations of heat transfer in pulsed thermography applied to porous CFRP", AIP conference proceedings vol. 1430, pp. 1025-1032, 2012.

[16] Stone D.E.W., Clarke B., "Ultrasonic attenuation as a measure of void content in carbon-fibre reinforced plastics", Non-Destructive Testing, vol. 8(3), pp. 137-145, 1975.

[17] Zalameda J.N., "Measured through-the-thickness thermal diffusivity of carbon fiber reinforced composite materials", Journal of Composites Technology and Research, vol. 21, pp. 98-102, 1999.

[18] Rolfes R, Hamerschmidt U., "Transverse thermal conductivity of CFRP laminates: A numerical and experimental validation of approximation formulae", Composites Sciences and Technology, vol. 54, pp. 45-54, 1995.

[19] Bison B.G., Marinett S., Mazzoldi A., Grinzato E., Bressan C., "Cross-comparison of thermal diffusivity measurements by thermal methods". Infrared Physics \& Technology, vol. 43, pp. 127-132, 2002.

[20] Parker W.J., Jenkins R.J., Butler C.P.L., Abbott G., "Flash method of determining thermal diffusivity, heat capacity and thermal conductivity". Journal of Applied Physics, vol. 32(9), pp. 1679-1684, 1961. 